\title{
GPPS-BJ-2019-0107
}

\section{Experimental study of the dynamic responses of an asymmetric two-dimensional Bunsen flame}

\author{
Yichen Wang \\ Key Laboratory for Thermal Science and \\ Power Engineering of Ministry of Education, \\ Department of Energy and Power Engineering, \\ Tsinghua University \\ yc-wang18@mails.tsinghua.edu.cn \\ Beijing, 100084, China
}

\author{
Chunyan Li \\ Key Laboratory for Thermal Science and \\ Power Engineering of Ministry of Education, \\ Department of Energy and Power Engineering, \\ Tsinghua University \\ licy0207@126.com \\ Beijing, 100084, China
}

\author{
Suhui Li \\ Key Laboratory for Thermal Science and \\ Power Engineering of Ministry of Education, \\ Department of Energy and Power Engineering, \\ Tsinghua University \\ lisuhui@tsinghua.edu.cn \\ Beijing, 100084, China
}

\author{
Min Zhu \\ Key Laboratory for Thermal Science and \\ Power Engineering of Ministry of Education, \\ Department of Energy and Power Engineering, \\ Tsinghua University \\ zhumin@mail.tsinghua.edu.cn \\ Beijing, 100084, China
}

\begin{abstract}
Mean transverse flow can affect azimuthal modes in annular combustions due to the effect of the mean transverse flow on the flame responses. This study measured the flame responses of an asymmetric Bunsen flame subjected to transverse and axial acoustic waves. An experimental rig was set up to provide simultaneous transverse and axial perturbations. The experimental results were compared with analytical models based on the linearized G-equation. The analytical and experimental results both show that the axial FTF has a low-pass characteristic and the transverse FTF has a band-pass characteristic. The results also show the linear superposition of transverse and axial FTFs. Cases with different transverse mean flows show that the transverse flame response is caused by asymmetric flame surfaces. Flame positions and flame surface areas obtained from a camera also confirm the flame response mechanism.
\end{abstract}

\section{INTRODUCTION}

Lean-premixed combustion technologies are used to reduce the emissions of gas turbines. However, the techniques frequently cause combustion instabilities in the gas turbines which can severely damage the combustion chambers (Lieuwen et al., 2005). Azimuthal thermoacoustic modes have been widely observed in the combustor because the circumference is longer than the length (O'Connor et al., 2015). Numerical (Staffelbach et al., 2009; Bauerheim et al., 2015a) and experimental (Worth and Dawson, 2013; Prieur et al., 2017) results have shown that the azimuthal instabilities shift randomly among standing, spinning and mixed modes.

A linear quasi-one dimensional model (Bauerheim et al., 2014; Bauerheim et al., 2015b) was developed to predict the azimuthal modes, which assumes the acoustic waves are one dimensional and uses Flame Transfer Functions (FTF) flame models. The model showed (Bauerheim et al., 2015b) that a mean azimuthal flow in the chamber can affect the azimuthal mode. The flame models used in the linear quasi-one dimensional model only show flame responses to the axial velocity perturbation. However, some experimental results (Bourgouin et al., 2015; Bourgouin, 2014) have shown that the flame is affected by both axial and azimuthal modes. The flame model should be improved to incorporate the transverse perturbation effects.

Experiments for a lab-scale annular chamber are useful to study the acoustic mode characteristics. Flame dynamics and heat release rate were studied (Dawson and Worth, 2014) for standing and spinning self-excited azimuthal modes. The heat release rate of each flame varied according to the location, acoustic mode and flame separation distance. Flame dynamics for different spinning directions (Nygård et al., 2019) were studied and a novel averaging method was used to decompose the standing mode into ACW and $\mathrm{CW}$ spinning components. Some results (Worth and Dawson, 2017) also proved that the equivalence ratio can strongly affect the azimuthal modes. 


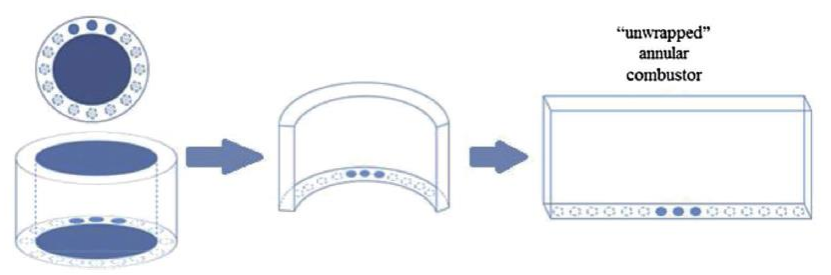

Fig. 1. An 'unwrapped' annular combustor (O'Connor et al., 2015)

Experiments are very expensive for a whole lab-scale annular chamber. Therefore, some experiments 'unwrapped' the annular chamber to a one-dimensional configuration as shown in Fig. 1. This configuration can be used to study the flame responses to axial and transverse perturbations. Several experiments (O'Connor and Lieuwen, 2012; Malanoski et al., 2014) found that the transverse acoustic wave can affect the vortex shedding. Another experiment (Hauser et al., 2010) applied simultaneous transverse and axial perturbations to a single swirl burner. The displacement of the heat release centre and rotational motion of flame are both affected by two-dimensional excitations.

In previous literature, most experiments have studied symmetric flame characteristics. However, when there is a mean transverse flow, the flame becomes asymmetric so that the transverse responses appear. An analytical flame model (Li et al., 2017) was developed to predict the asymmetry effect. The model was developed from a G-equation model (Schuller et al., 2003) assuming small velocity perturbations. Further experimental studies are needed to confirm the asymmetry flame response to transverse and axial perturbations. In this paper, an experimental system was set up to achieve simultaneous transverse and axial perturbations. The flame responses were studied for various mean transverse flows and the FTFs were calculated for various frequencies. The flame surface motion was captured by a high speed camera to study the flame response mechanism. This paper will show the flame dynamics for two-dimensional perturbations and the flame response mechanism of laminar Bunsen flame.

\section{METHODOLOGY}

An analytical method based on G-equation is used to simulate the flame position and FTFs and experiments were conducted to verify the analytical model.

\section{Analytical method}

\section{Analytical model}

An asymmetric Bunsen flame is shown in Fig. 2. The axial and transverse flow velocities are written as $\vec{v}=$ $(u, v)=\left(\bar{u}+u^{\prime}, \bar{v}+v^{\prime}\right)$, which can be divided into mean and perturbation parts.

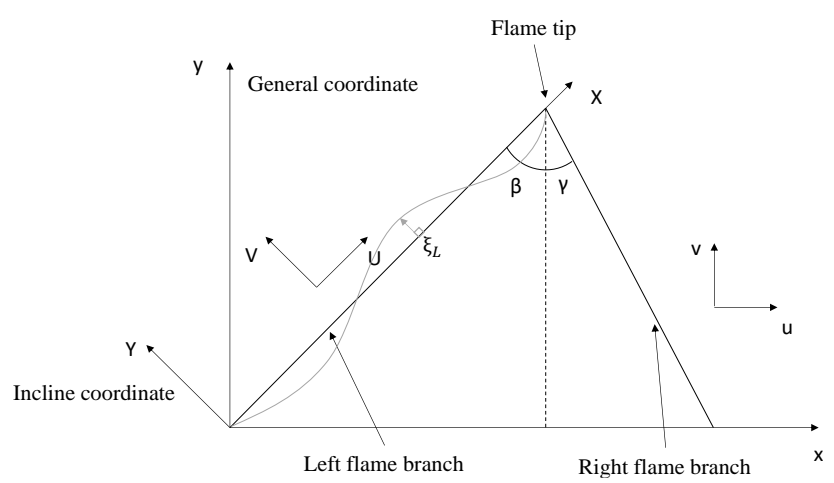

Fig. 2. Schematic of two-dimensional Bunsen flame

The flame surface position can be obtained by solving the G-equation which is

$$
\frac{\partial G}{\partial t}+\vec{v} \nabla G=S_{L}|\nabla G|
$$

in which $S_{L}$ represents the flame speed. The $\mathrm{G}<0$ means the gas is unburnt, $G>0$ means the gas is burnt and $G=0$ is the flame position. The flame position is assumed to make small perturbations near the mean flame position. Therefore, an incline coordinate is established along each flame branch to simplify the analysis. In the incline coordinate, the Gequation is,

$$
-\frac{\partial \xi}{\partial t}-U \frac{\partial G}{\partial X}+V=S_{L}\left[\left(\frac{\partial \xi}{\partial X}\right)^{2}+1\right]^{\frac{1}{2}}
$$

where the $\xi$ is the flame position in the inclined coordinate. Assuming the perturbations and velocities are linear, small and harmonic, that is,

$$
\begin{aligned}
\xi(X, t) & =\tilde{\xi}(X) e^{-i \omega t} \\
V^{\prime}(X, t) & =\tilde{V}(X) e^{-i \omega t}
\end{aligned}
$$

then the solution of left flame branch is

$$
\xi_{L}(x)=\frac{V_{L}^{\prime} R}{i \omega^{*}}\left[1-\exp \left(i \omega^{*} \frac{x}{R} \frac{1}{B_{L}}\right)\right] e^{-i \omega t}
$$

where $\quad B_{L}=\left(s_{u} \sin \beta+s_{v} \cos \beta\right) \sin \beta \quad$ and $V_{L}^{\prime}=$ $s_{u 1} \cos \beta-s_{v 1} \sin \beta$. The frequency and velocities are normalized as $\omega^{*}=\omega R / S_{L}, s_{u 1}=\tilde{u} / S_{L}, s_{v 1}=\tilde{v} / S_{L}$, $s_{u}=\bar{u} / S_{L}, s_{v}=\bar{v} / S_{L}$, and $S_{L}$ means the flame speed. The flame position in the inclined coordinate should be transformed into the general coordinate for further calculation. The flame position in general coordinate can be written as,

$$
y_{L}(x, t)=\frac{x}{\tan \beta}+\frac{\mathrm{R}\left[\xi_{L}(x, t)\right]}{\sin \beta}
$$

where small perturbation assumption is used to simplify the transformation relationship. In the similar way, the solution of right flame branch is obtained as

$$
\xi_{R}(x)=\frac{V_{R}^{\prime} R}{i \omega^{*}}\left[1-\exp \left(i \omega^{*} \frac{2 R-x}{R} \frac{1}{B_{R}}\right)\right] e^{-i \omega t}
$$




$$
y_{R}(x, t)=\frac{2 R-x}{\tan \gamma}+\frac{\mathrm{R}\left[\xi_{L}(x, t)\right]}{\sin \gamma}
$$

where, $B_{R}=\left(s_{v} \sin \gamma-s_{u} \cos \gamma\right) \sin \gamma$ and $V_{R}^{\prime}=$ $-s_{u 1} \cos \gamma-s_{v 1} \sin \beta$. Then, the FTF is integrated from the flame surface area as,

$$
\begin{gathered}
A_{L / R}=\int \sqrt{1+\left(\frac{\partial y_{L / R}}{\partial x}\right)^{2}} d x \\
A(t)=A_{L}(t)+A_{R}(t)
\end{gathered}
$$

The range of integration is determined by flame tip position and the size of burner. The flame tip position can be obtained by solving the equation

$$
y_{L}\left(x_{t i p}, t\right)=y_{R}\left(x_{t i p}, t\right)
$$

which can be simplified as,

$$
\frac{x_{t i p}}{\tan \beta}+\frac{V_{L}^{\prime} R}{\omega^{*} \sin \beta} P_{L}=\frac{2 R-x_{t i p}}{\tan \gamma}+\frac{V_{R}^{\prime} R}{\omega^{*} \sin \gamma} P_{R}
$$

where $P_{L}$ and $P_{R}$ are related to time and flame tip abscissa,

$$
\begin{gathered}
P_{L}=\sin (\omega t)+\sin \left(\frac{\omega}{\bar{U}_{L}} \frac{x_{t i p}}{\sin \beta}-\omega t\right) \\
P_{R}=\sin (\omega t)+\sin \left(\frac{\omega}{\bar{U}_{R}} \frac{2 R-x_{t i p}}{\sin \gamma}-\omega t\right)
\end{gathered}
$$

Equation 7 can only be solved by numerical method so that the flame surface area is obtained by numerical integral. The flame surface area is processed by Fourier transform and divided by the velocity perturbation amplitude for FTFs.

\section{Analytical results and discussion}

The analytical model can show some characteristics of the Bunsen flame for various cases.

For the transverse FTF (Trans FTF ), the results show that it has a band-pass characeristic. Trans FTFs with various mean transverse flow are shown in the Fig. 3. The amplitudes of Trans FTFs increase with the mean transfer flow, which means that the transverese mean flow leads to the transverse responses. Trans FTFs with various axial flow are shown in Fig. 4. The amplitudes decrease with increasing axial flow. When axial flow increase, the flame become more symmetric. Therefore, the asymmetry of flame is the source the transverse flame responses.
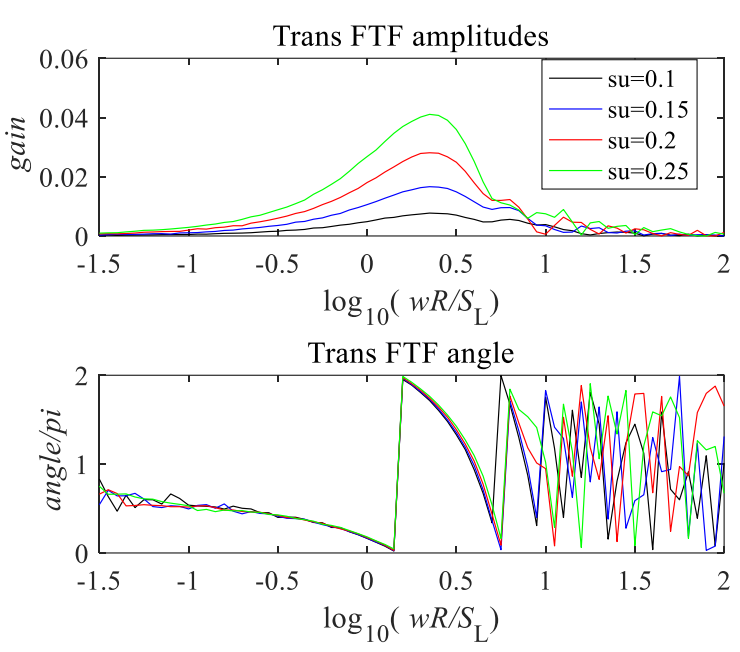

Fig. 3.Transverse FTFs for various transverse mean flow, parameters: $s_{u 1}=0.1, s_{v 1}=0, s_{v}=1.25$
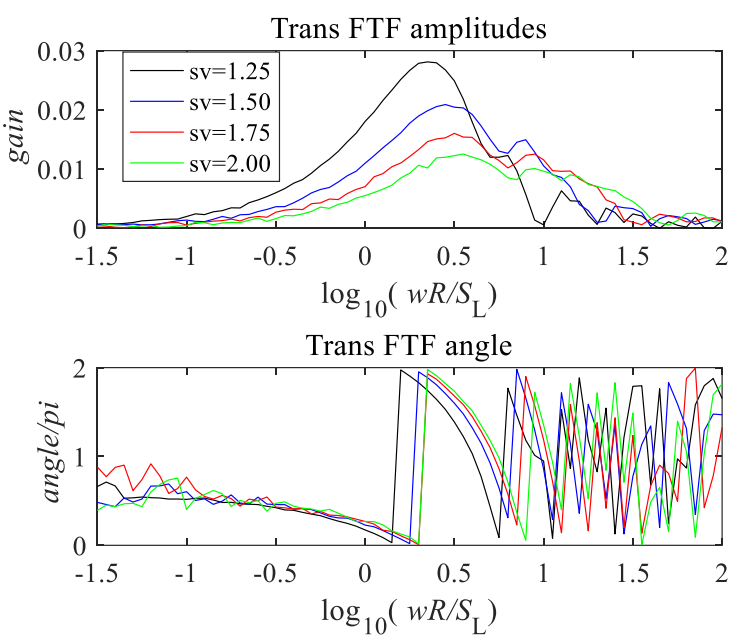

Fig. 4. Transverse FTFs for various axial mean flow, parameters: $s_{u 1}=0.1, s_{v 1}=0, s_{u}=0.2$
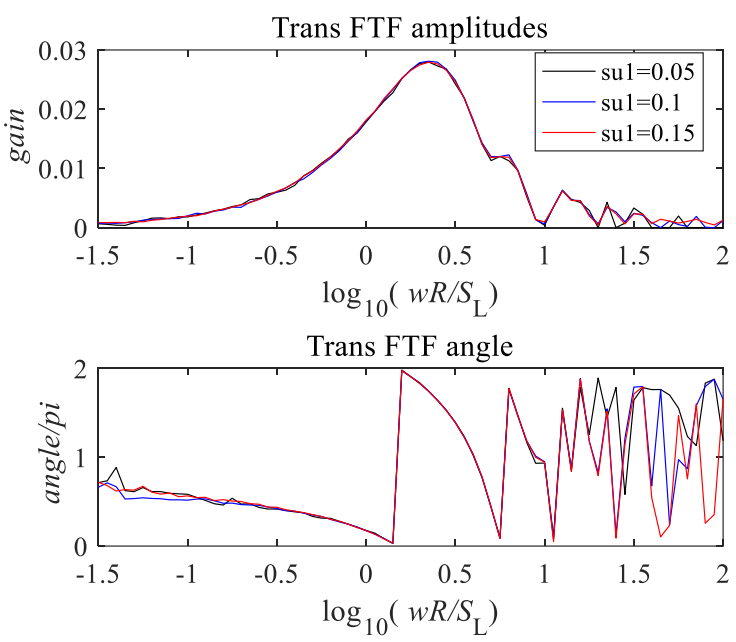

Fig. 5. Transverse FTFs for various transverse perturbation amplitudes,

parameters: $s_{v 1}=0, s_{u}=0.2, s_{v}=1.25$ 
For axial FTFs, the results show a low-pass characteristic. Figure 6 compares the axial FTFs for various axial mean flow, and Fig.7 compares the axial FTFs for various transverse mean flow. They show that the mean flow has little effect on the axial FTFs. The transverse FTFs for various axial perturbation amplitudes are shown in the Fig. 5 and the axial FTFs for various amplitudes are shown in Fig. 8. The results show that the FTFs are linear when the perturbations are small.
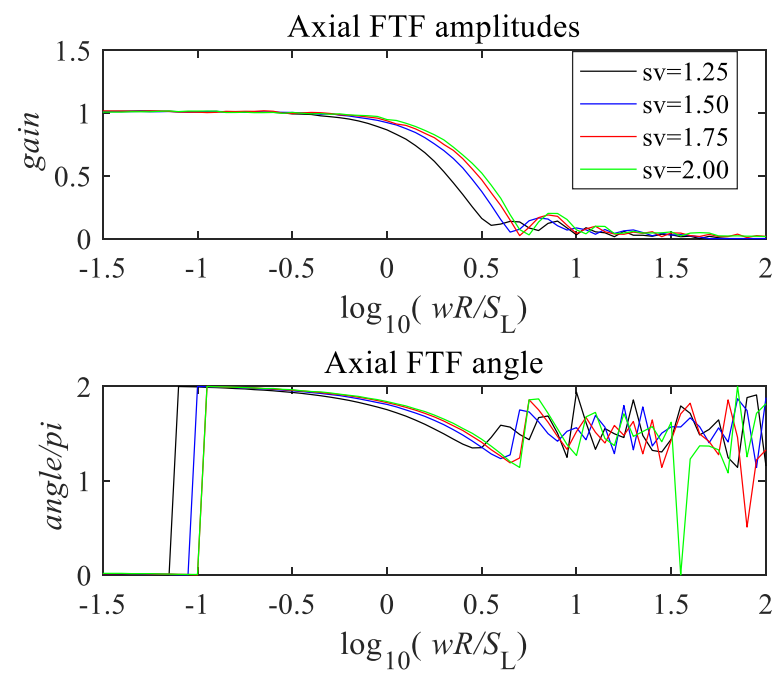

Fig. 6. Axial FTFs for various axial mean flow, parameters: $s_{u 1}=0, s_{v 1}=0.05, s_{u}=0.2$
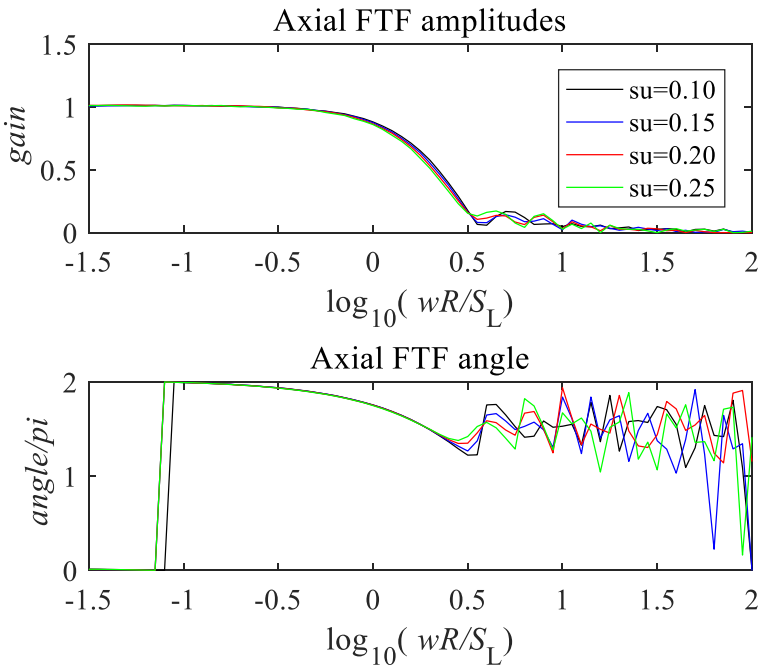

Fig. 7. Axial FTFs for various transverse mean flow, parameters: $s_{u 1}=0, s_{v 1}=0.05, s_{v}=1.25$
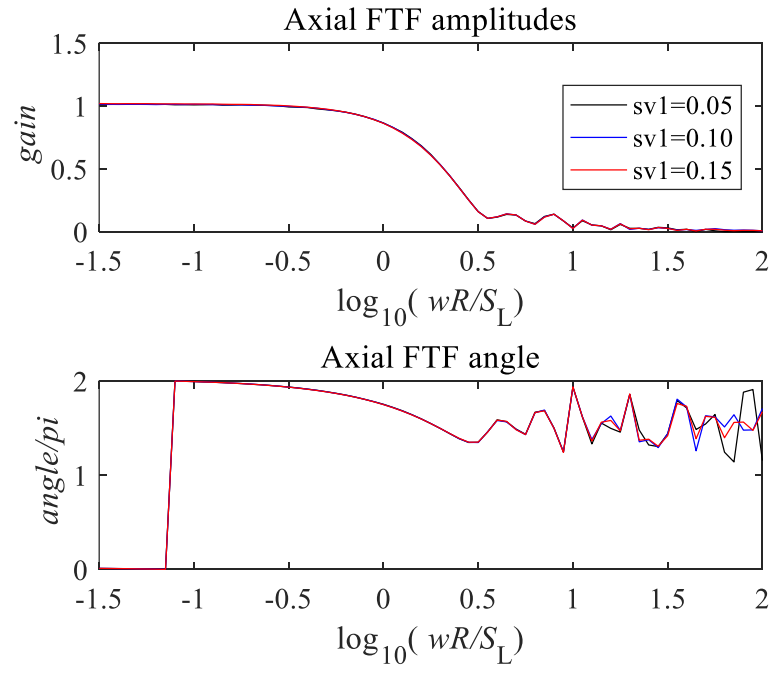

\section{Fig. 8. Axial FTFs for various axial perturbation amplitudes,}

parameters: $s_{u 1}=0, s_{u}=0.2, s_{v}=1.25$

\section{Experimental method}

An experimental rig was used to measure the axial and transverse flame disturbance. The whole experimental rig was designed as $\mathrm{T}$ shape and divided into a combustion test section and an inlet section as shown in Fig. 9. The fuel and air were. mixed in the inlet section and went through a 320 mm square channel used to stabilize the flow rate. The burner outlet was $10 \mathrm{~mm} \times 60 \mathrm{~mm}$ to form a twodimensional Bunsen flame. The combustion test section was a $2 \mathrm{~m}$ long extended pipe. The cross sectional area was 150 $\mathrm{mm} \times 90 \mathrm{~mm}$, which satisfies the plane wave condition. The upstream of the transverse pipe had a $500 \mathrm{~mm}$ long aluminium honeycomb pipe to stabilize the mean transverse flow. There were holes above the burner outlet for the exhaust and a porous honeycomb ceramic was fitted to reduce acoustic wave leakage. Quartz glasses were set on both sides to measure the heat release rates and dynamic processes. Two loudspeakers were set at the inlet section to produce axial disturbance while four loudspeakers were set at the transverse pipe to adjust the transverse disturbance.

The heat release rate and pressures were measured using Zolix Omni- $\lambda 300$ to calculate the axial and transverse FTFs. The pressures were measured using four B\&K 4938-A-011 microphones and two G.R.A.S. microphones. The microphone sensitivities were $10 \mathrm{mV} / \mathrm{Pa}$ with an error of $\pm 3 \mathrm{~dB}$. All the data were collected using a PXI system manufactured by the NI Company. The system contained a PXIe-082 chassis, a PXIe-8108 controller and a PXIe-4496 dynamic signal analyser. 


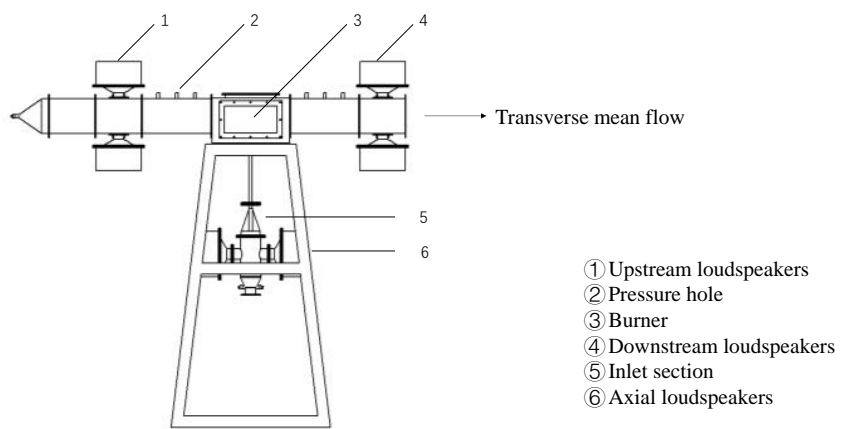

Fig. 9. Two-dimensional flame experimental rig

The dynamic processes were captured using a high speed camera. The camera was a Phantom-V210 manufactured by Vision Research Company. The maximum resolution of the high speed camera was $1024 * 800$ and maximum exposure rate was 2000 frames per second. The flame in Fig. 10 was shot at 720 fps. 60 pictures were shot from the same phase position of various periods and averaged to produce one picture.
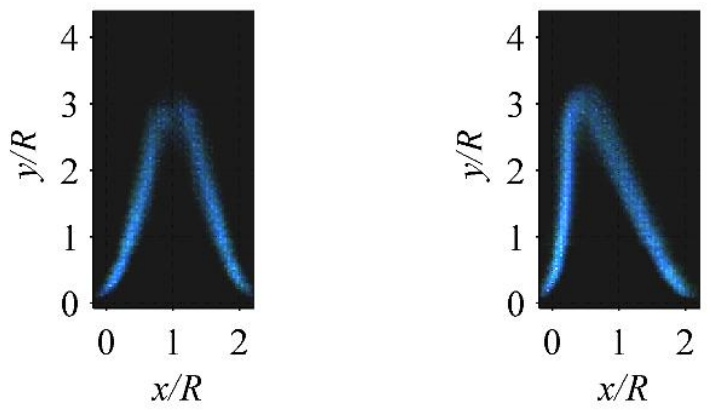

Fig. 10. Symmetric and asymmetric Bunsen flame

\section{RESULTS AND DISCUSSION}

\section{Experiment cases}

The experiment cases contain four parameters, which are mean transverse flow, perturbation frequency, transverse perturbation amplitude and axial perturbation amplitude.

The fuel used is natural gas and the mixture is lean premixed for all the cases. In the experimental measurement, the first step is to measure the axial flame responses for various axial perturbation amplitudes. The results confirm that the axial flame response is in a linear range. Then the flame responses under two-dimensional perturbations are measured. The flow velocities at the burner can be obtained by using the two microphone method. Therefore, the transverse flame responses can be obtained by using the equation

$$
\hat{q}=F T F_{a} \hat{v}+F T F_{t} \hat{u}
$$

in which $\mathrm{FTF}_{\mathrm{a}}$ means axial FTF and $\mathrm{FTF}_{\mathrm{t}}$ means transverse FTF. The results obtained under different amplitudes coincided with each other, which showed that the flame responses for two-dimensional perturbations have superposition effect.

The experiment figures in the paper are phase averaged results from 40-period pictures. And the thickness of flame is around $1.5 \mathrm{~mm}$. The medium line of flame is compared with the analytical results

\section{Axial FTFs}

The axial FTFs are shown in the Fig. 11 Figure 11(a) shows the axial FTF of a symmetric flame. Figure 11(b) and (c) shows the axial FTF of asymmetric flames.

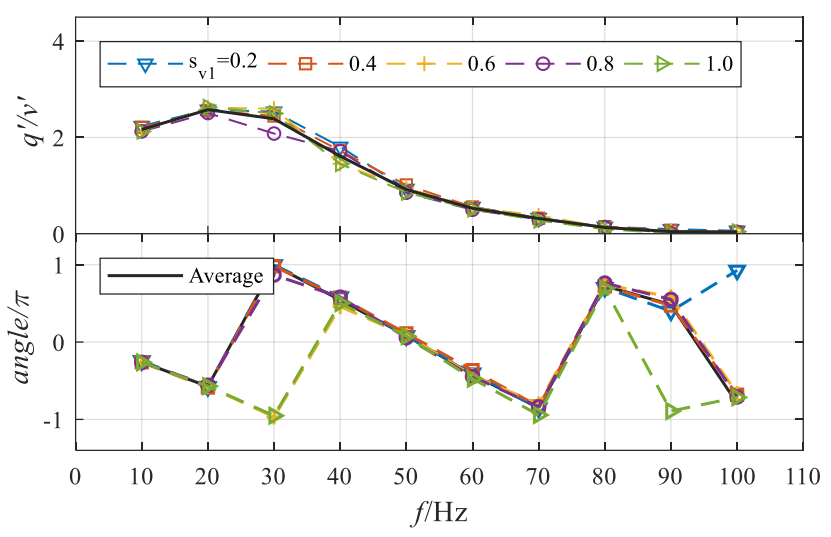

(a) Transverse mean flow: $u=0 \mathrm{~m} / \mathrm{s}$

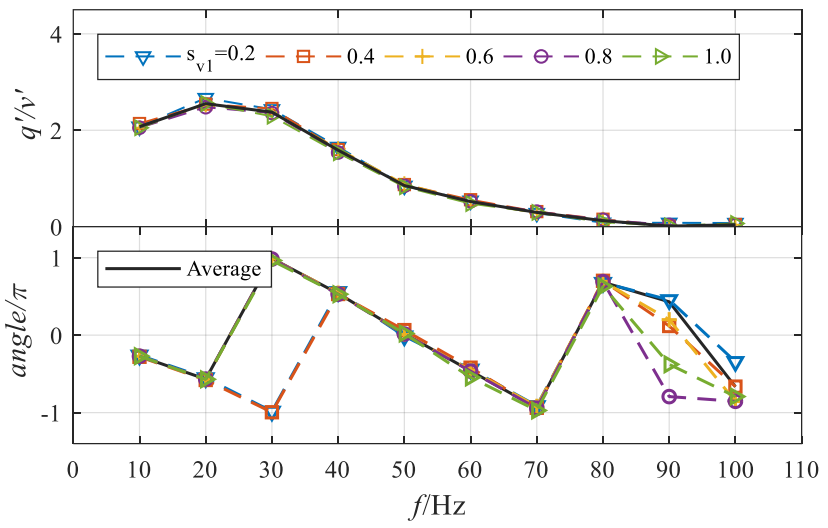

(b) Transverse mean flow: $u=0.04 \mathrm{~m} / \mathrm{s}$

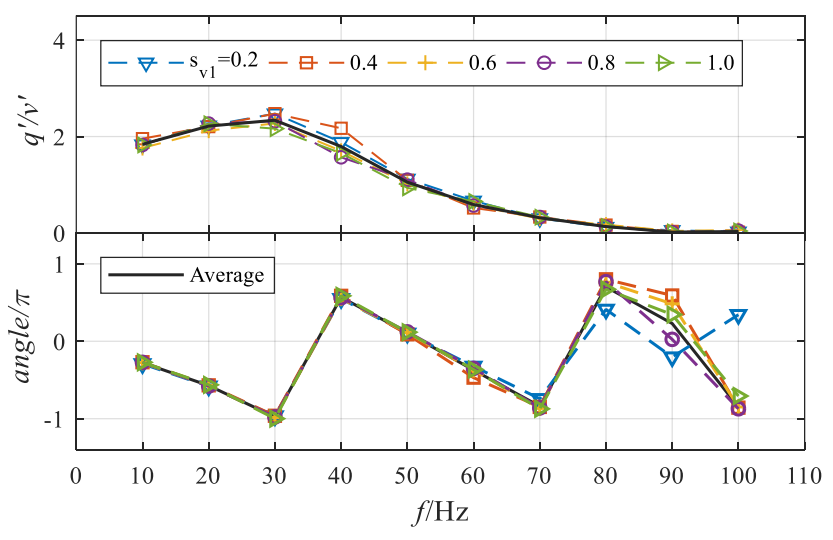

(c) Transverse mean flow: $u=0.06 \mathrm{~m} / \mathrm{s}$

\section{Fig. 11. Axial FTFs for various mean transverse flows, parameter: $v=0.62 \mathrm{~m} / \mathrm{s}$}

The results show that the axial FTFs have a low-pass characteristic. The asymmetry of flame has little effect on the 
axial FTF. The results of different amplitude cases show that the FTF is located in the linear range, which means the perturbation amplitudes will not affect the FTF.

The analytical model has a good agreement with the experimental results. The dynamic processes of flame are captured by high speed camera and compared with the analytical results. The comparisons are shown in the Fig. 12 for a symmetric flame, and Fig. 13 for an asymmetric flame.

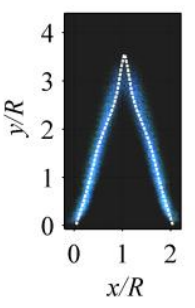

(a) 0

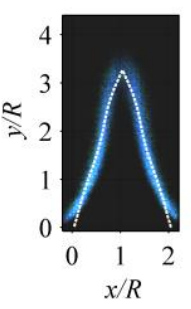

(d) $\pi$

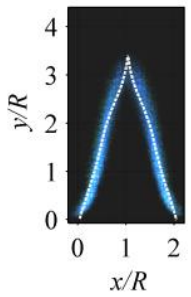

(b) $\pi / 3$

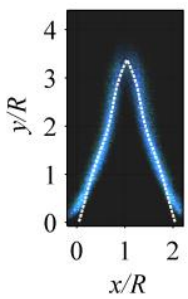

(e) $4 \pi / 3$

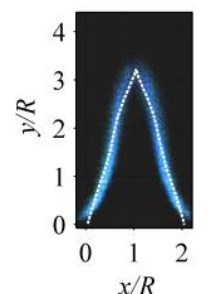

(c) $2 \pi / 3$

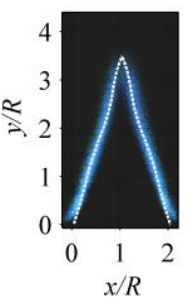

(f) $5 \pi / 3$
Fig.12 Comparison between experimental and analytical results, a symmetry flame, perturbation frequency: $60 \mathrm{~Hz}$

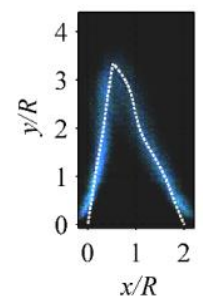

(a) 0

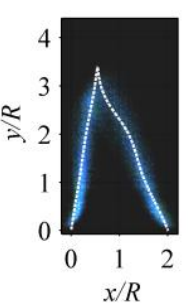

(d) $\pi$

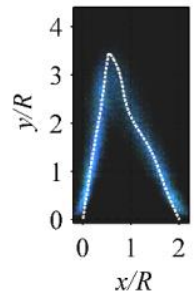

(b) $\pi / 3$

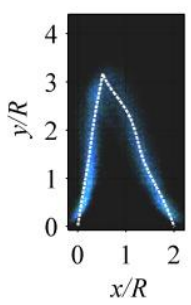

(e) $4 \pi / 3$

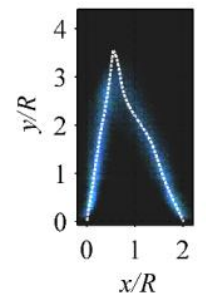

(c) $2 \pi / 3$

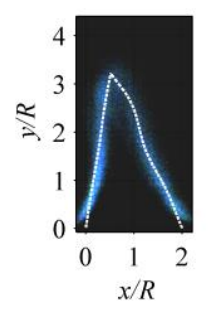

(f) $5 \pi / 3$
Fig.13 Comparison between experimental and analytical results, an asymmetry flame, perturbation frequency: $60 \mathrm{~Hz}$

Both figures show the flame positions in a perturbation period. The analytical model can accurately track the flame positions under the axial perturbations. Stretch rate and heat loss affect the flame position at flame tip and flame root where there are some distinctions. For asymmetric flame, the right branch has more wrinkles than the left branch due to larger angle between right branch and the axial flow direction.

\section{Transverse FTFs}

The transverse FTFs are shown in the Fig. 14 Figure 14(a) shows the transverse FTF of a symmetric flame. Figure 14(b) shows the transverse FTF of an asymmetric flame.

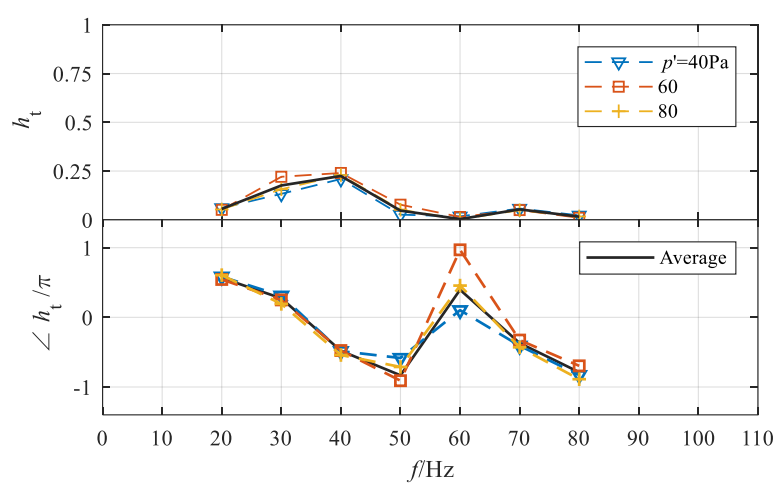

(a) $\mathrm{u}=0 \mathrm{~m} / \mathrm{s}$

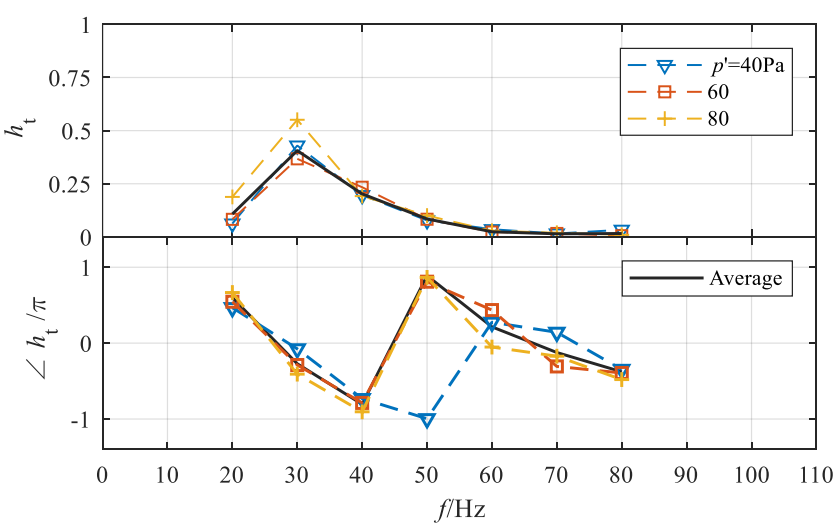

(b) $u=0.04 \mathrm{~m} / \mathrm{s}$

Fig. 14. Transverse FTFs for various mean transverse flows parameters: $v=0.62 \mathrm{~m} / \mathrm{s}$

The transverse FTFs are calculated by subtracting axial responses from the two-dimensional responses. The coincidence for various perturbation amplitudes shows that the superposition effect of axial and transverse FTFs.

The results show that the transverse FTFs have a bandpass characteristic. The asymmetry of flame is the source of transverse responses. The dynamic processes of flame are shown in the Fig. 15 for a symmetric flame, and Fig. 16 and 17 for an asymmetric flame for different perturbation frequencies. All the figures actually capture the flame dynamics for two-dimensional perturbations. 


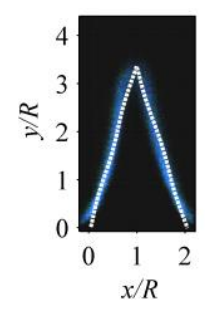

(a) 0

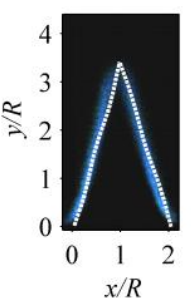

(d) $\pi$

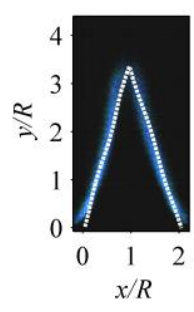

(b) $\pi / 3$

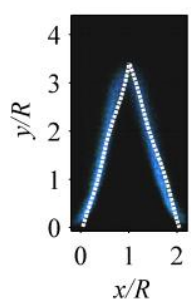

(e) $4 \pi / 3$

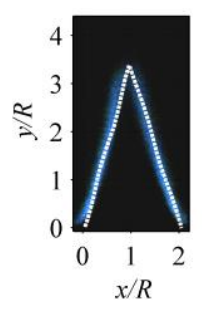

(c) $2 \pi / 3$

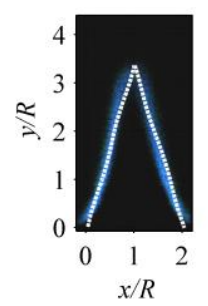

(f) $5 \pi / 3$
Fig. 15. Comparison between experimental and analytical results, a symmetry flame, perturbation frequency: $60 \mathrm{~Hz}$

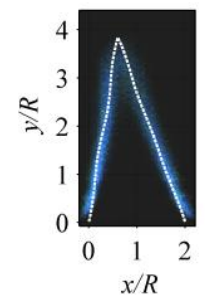

(a) 0

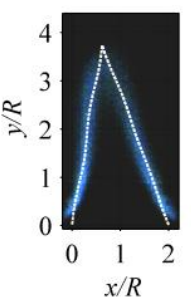

(d) $\pi$

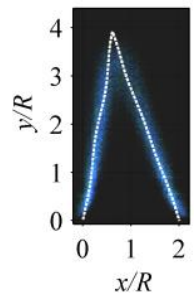

(b) $\pi / 3$

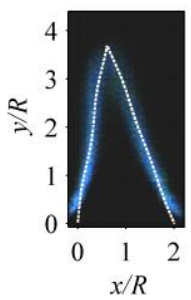

(e) $4 \pi / 3$

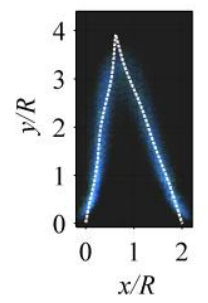

(c) $2 \pi / 3$

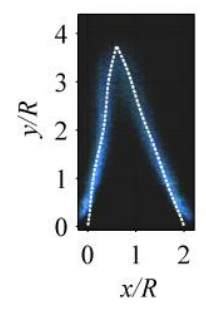

(f) $5 \pi / 3$
Fig. 16. Comparison between experimental and analytical results, an asymmetry flame, perturbation frequency: $60 \mathrm{~Hz}$

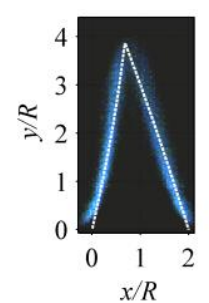

(a) 0

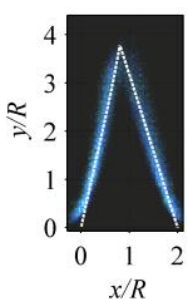

(b) $\pi / 3$

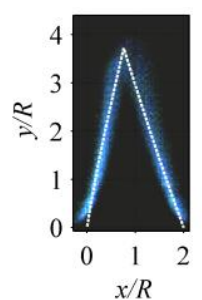

(c) $2 \pi / 3$

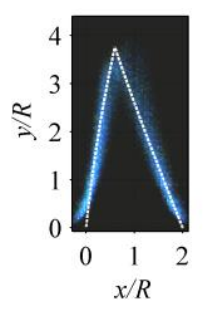

(d) $\pi$

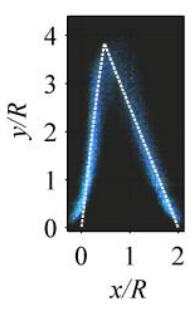

(e) $4 \pi / 3$

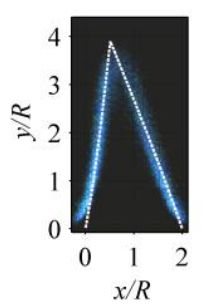

(f) $5 \pi / 3$

Fig. 17. Comparison between experimental and analytical results, an asymmetry flame, perturbation frequency: $10 \mathrm{~Hz}$

For a symmetric flame, the positions of the left and right branches of the flame are almost symmetrical so that the transverse response disappears. For an asymmetric flame at $60 \mathrm{~Hz}$, the position of left and right branches differ so that the transverse response appear. The analytical model can capture the motion of wrinkles which the left branch has more than the right branch. The reason is that for the right branch, the effect of transverse perturbations is offset by the induced axial perturbation. More wrinkles lead to little change of the mean flame surface area so that right branch is the source of flame surface area change. The motion of flame is more clearly shown in Fig. 18. For an asymmetric flame at $10 \mathrm{~Hz}$, both left and right branch has little fold because the acoustic wavelength is much longer than the flame length. Both branches act as straight lines rotate on their attachment points. The change of one flame surface area is offset by the other. Therefore, the flame at low frequency also has little transverse response.

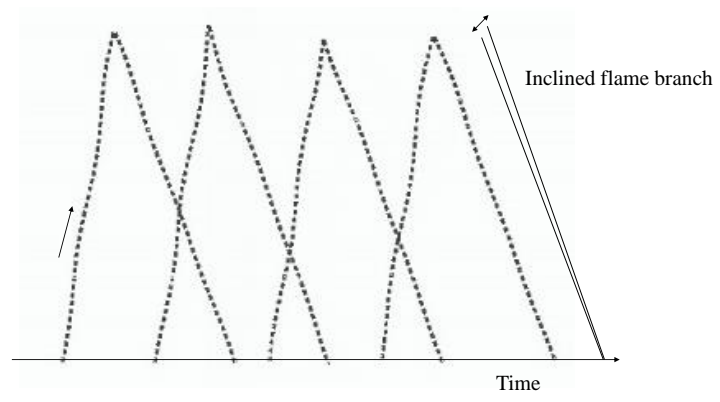

Fig. 18. Motion of flame, an asymmetry flame, perturbation frequency: $60 \mathrm{~Hz}$

\section{CONCLUSIONS}

The experimental results show that the axial FTF has a low-pass characteristic and the transverse FTF has a bandpass characteristic The superposition effect is also proved. The analytical model is verified by comparison with experimental results. The cases with different transverse mean flow show that the transverse flame response is caused by asymmetric flame surface. The asymmetry between left and right branches is the source of transverse responses. The fold structure is also important for the two-dimensional responses, which is related to acoustic wavelength and mean flame positions. 


\section{NOMENCLATURE}

\begin{tabular}{|c|c|}
\hline $\mathrm{FTF}_{\mathrm{a}} / \mathrm{FTF}_{\mathrm{t}}$ & axial/ transverse flame transfer function \\
\hline$A_{L / R}$ & flame surface area of each branch \\
\hline$B_{L} / B_{R}$ & $\begin{array}{l}\text { scaled mean velocity of left /right } \\
\text { branch }\end{array}$ \\
\hline$P_{L} / P_{R}$ & $\begin{array}{l}\text { flame position perturbation composition } \\
\text { of left/ right branch }\end{array}$ \\
\hline$s_{u}=\bar{u} / S_{L}$ & normalized transverse mean flow \\
\hline$s_{u 1}=\tilde{u} / S_{L}$ & normalized transverse perturbation \\
\hline$s_{v}=\bar{v} / S_{L}$ & normalized axial mean flow \\
\hline$s_{v 1}=\tilde{v} / S_{L}$ & normalized axial perturbation \\
\hline$S_{L}$ & laminar flame speed \\
\hline$u, v$ & velocities in general coordinate \\
\hline$U, V$ & velocities in incline coordinate \\
\hline$V_{L}^{\prime} / V_{R}^{\prime}$ & $\begin{array}{l}\text { scaled forcing amplitude of left/ right } \\
\text { branch }\end{array}$ \\
\hline$\xi_{L} / \xi_{R}$ & $\begin{array}{l}\text { left/ right flame branch position in the } \\
\text { incline coordinate }\end{array}$ \\
\hline$\omega^{*}=\omega R / S_{L}$ & normalized angular frequency \\
\hline
\end{tabular}

\section{REFERENCES}

Bauerheim M., Cazalens M., and Poinsot T. (2015). A theoretical study of mean azimuthal flow and asymmetry effects on thermo-acoustic modes in annular combustors. Proceedings of the Combustion Institute, 35 (3), 3219-3227. doi: 10.1016/i.proci.2014.05.053

Bauerheim M., Parmentier J. F., Salas P., Nicoud, F., and Poinsot T. (2014). An analytical model for azimuthal thermoacoustic modes in an annular chamber fed by an annular plenum. Combustion and Flame, 161 (5), 1374-1389. doi: 10.1016/j.combustflame.2013.11.014

Bauerheim M., Staffelbach G., Worth N. A., Dawson J. R., Gicquel L. Y. M. and Poinsot T. (2015). Sensitivity of lesbased harmonic flame response model for turbulent swirled flames and impact on the stability of azimuthal modes. Proceedings of the Combustion Institute, 35 (3), 3355-3363. doi: 10.1016/j.proci.2014.07.021

Bourgouin J. F. (2014). Characterization and modeling of a spinning thermoacoustic instability in an annular combustor equipped with multiple matrix injectors. Journal of Engineering for Gas Turbines and Power 137 (2) 021503021503-021511 doi:10.1115/1.4028257

Bourgouin J. F., Durox D., Moeck J. P., Schuller T., an d Candel S. (2015). A new pattern of instability observe $\mathrm{d}$ in an annular combustor: the slanted mode. Proceedin gs of the Combustion Institute, 35(3), 3237-3244. doi: 1 $\underline{0.1016 / j . p r o c i .2014 .06 .029}$
Dawson J. R. and Worth N. A. (2014). Flame dynamics and unsteady heat release rate of self-excited azimuthal modes in an annular combustor. Combustion and Flam e, 161 (10), 2565-2578. doi: 10.1016/j.combustflame.201 $\underline{4.03 .021}$

Hauser M., Lorenz, M. and Sattelmayer T. (2010). Influence of transversal acoustic excitation of the burner approach flow on the flame structure. Journal of Engineering for Gas Turbines and Power, 133 (4), 041501-041501-8. doi: $\underline{10.1115 / 1.4002175}$

Li, C., Zhu, M. and Moeck J. P. (2017). An analytical study of the flame dynamics of a transversely forced as ymmetric two-dimensional bunsen flame. Combustion Th eory and Modelling, 21 (5), 976-995 doi: 10.1080/13647 $\underline{830.2017 .1327677}$

Lieuwen T., Yang Vigor and Lu Frank K. (2005). Combustion instabilities in gas turbine engines operational experience, fundamental mechanisms and modeling, Reston, Va.: American Institute of Aeronautics and Astronautics.

Malanoski M., Aguilar M., Shin D. H., and Lieuwen, T. (2014). Flame leading edge and flow dynamics in a swirling, lifted flame. Combustion Science and Technology, 186 (12), 1816-1843. doi: 10.1080/00102202.2014.923410

Nygård Håkon T., Mazur M., Dawson J. R. and Worth N. A. (2019). Flame dynamics of azimuthal forced spinning and standing modes in an annular combustor. Proceedings of the Combustion Institute, 37 (4), 5113-5120. doi: 10.1016/j.proci.2018.08.034

O'Connor J. and Lieuwen T. (2012). Recirculation zone dynamics of a transversely excited swirl flow and flame. Physics of Fluids, 24 (7), 293-364. doi: 10.1063/1.4731 $\underline{300}$

O'Connor J., Acharya V. and Lieuwen T. (2015). Transverse combustion instabilities: acoustic, fluid mechanic, and flame processes. Progress in Energy and Combustion Science, 49, 1-39. doi: 10.1016/j.pecs.2015.01.001

Prieur K., Durox D., Schuller T., and Candel S. (2017). A hysteresis phenomenon leading to spinning or standin $\mathrm{g}$ azimuthal instabilities in an annular combustor. Combu stion and Flame, 175, 283-291. doi: 10.1016j.combustion flame.2016.05.021

Schuller T., Durox D., and Candel S. (2003). A unified model for the prediction of laminar flame transfer functi ons: comparisons between conical and $\mathrm{V}$-flame dynamic s. Combustion and Flame, 134 (1), 21-34. doi: 10.1016/ S0010-2180(03)00042-7

Staffelbach G., Gicquel L. Y. M., Boudier G., and Poinsot T., (2009). Large eddy simulation of self excited azimuthal modes in annular combustors. Proceedings of the Combustion Institute 32 (2), 2909-2916. doi: 10.1016/j.proci.2008.05.033

Worth N. A. and Dawson J. R. (2013). Modal dynamics of self-excited azimuthal instabilities in an annular combustion chamber. Combustion and Flame, 160 (11), 2476-2489. doi: 10.1016/j.combustflame.2013.04.031

Worth N. A., and Dawson J. R. (2017). Effect of equivalence ratio on the modal dynamics of azimuthal combustion instabilities. Proceedings of the Combustion Institute, 36 (3), 3743-3751. doi: 10.1016/j.proci.2016.06.115 